\section{Air capture update}

To the Editor - The world is struggling to reduce greenhouse-gas emissions through conventional mitigation, and initially high expectations for the United Nations Climate Change Conference (Copenhagen, 7-18 December) are seemingly being scaled back almost daily. It thus seems that brute force efforts to remove and sequester carbon dioxide, such as air capture, will come to occupy an ever greater role in climate-policy discussions. Air capture refers to the direct removal and sequestration of carbon dioxide from ambient air. Investigations of the technologies that would remove this carbon dioxide, along with the associated costs and benefits, are attracting growing attention.

In recent months, several reports have endorsed looking at air capture more closely. A comprehensive assessment concluded that there was no doubt air-capture technologies could be developed, although questions regarding cost and the subsequent sequestration of the carbon dioxide will need to be addressed ${ }^{2}$. Indeed, at least according to an idealized assessment, the costs are comparable to more conventional routes of mitigation ${ }^{3}$, and it is likely that costs will fall in the future ${ }^{2}$. Most discussion of air capture centres on chemical removal methods, but there have been other proposals for biological and geological options. A sorbent-based chemical removal method, and another, using algae to convert carbon dioxide to organic carbon, could also be viable means of carbon capture: a recent report ${ }^{1}$ concluded that such methods would be preferable to geoengineering through management of solar radiation because of the smaller risk of unintended consequences.

The primary uncertainties surrounding air capture stem more from the lack of large-scale testing rather than scientific or technical concerns ${ }^{4}$. There are no more than two dozen researchers studying air capture and only US $\$ 1.5$ million of government funding available worldwide ${ }^{5}$. There is little point in debating specific details about costs or large-scale feasibility until demonstration projects are undertaken ${ }^{5}$.

There are other possible methods for air capture: natural reactions that trap carbon dioxide in volcanic rock could be enhanced ${ }^{7}$. A recent provocative study ${ }^{6}$ argues that irrigating the deserts of the Sahara and Australian outback could create forests, and thus biologically sequester most global emissions from fossil fuels for many decades or longer.

Given increasing concerns that many countries will be unable to make the emissions cuts deemed necessary by governments, air capture may become a last resort for climate mitigation. Governments should help accelerate discussion and implementation by supporting air-capture projects, especially demonstration projects.

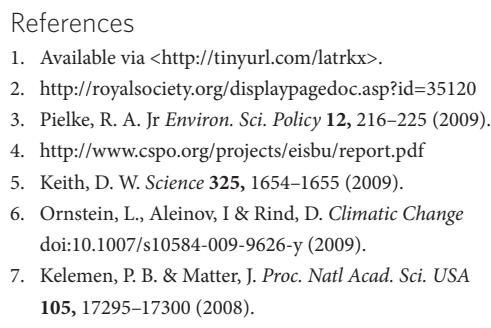

Roger Pielke Jr

University of Colorado, 1333 Grandview

Avenue, UCB 488, Boulder, Colorado

80309-0488, USA.

e-mail: pielke@colorado.edu

\title{
Energy for air capture
}

To the Editor - The use of air capture of carbon dioxide as a method for mitigating climate change is gaining traction in the debate surrounding geoengineering ${ }^{1-3}$. The potential of air capture is generally assessed in terms of monetary cost, estimated to be about a few hundred dollars per ton of carbon $^{1,4}$, leading to the suggestion that the costs of air capture are equal to, or even more favourable than, the costs of conventional mitigation efforts ${ }^{4}$.

However, the ultimate physical constraint on air capture is the energy required to power the systems. At present it takes 30-60 GJ to chemically remove one ton of carbon from the atmosphere ${ }^{5-7}$, comparable to the 40-70 GJ of energy generated during the production of one ton of emissions. Thus, using today's technology, it takes at least $\sim 0.5$ J of energy to capture the emissions generated in producing $1 \mathrm{~J}$ of fossil fuel energy. If the energy for capturing carbon comes from fossil fuels, then at least a third of society's fossil fuel energy would have to be diverted to air capture to eliminate all emissions. Alternatively, it would require an increase in the total production of fossil fuel energy of at least a third.

Neither option is impossible, but neither is definitively attainable, and both would be expensive and painful for the public. And by no means is either option obviously easier or cheaper than conventional mitigation. Technological breakthroughs may decrease the amount of energy required for air capture, but even capture energies of 20 and 10 GJ per ton of carbon require significant diversion of energy to air capture.

Renewable energy could be an option. Given typical estimates of mid-twentyfirst-century emissions of 15 billion tons of carbon per year, more than $14 \mathrm{TW}$ of renewable energy would be required to capture these emissions. This is comparable to total world energy consumption today. Although it may be possible to build this much renewable energy, would it make any sense to use it for air capture? It may be more prudent to simply replace the fossil fuel energy with renewable energy, which would lead to the about the same reduction in emissions.
There are clear benefits to air capture, and further research into this approach is clearly warranted. But at present the enormous amount of energy required for air capture represents a fundamental constraint to this approach, and it may preclude the possibility of using it to offset the majority of human emissions. Future analyses of air capture must explicitly take energy constraints into account.

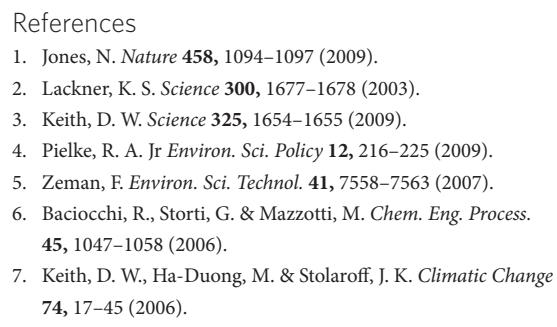

Andrew Dessler

Department of Atmospheric Sciences, Texas A\&M University, TAMU 3150, College Station, Texas 77843-3150, USA.

e-mail: adessler@tamu.edu 